Other radioisotopes, such as rhenium-188, are also starting to come into favor. Rhenium-188 is a beta emitter, like lutetium-177. But because it radiates at least twice as far into surrounding tissues, "it has a better chance of penetrating the interior of a solid tumor," says Chris Adams, founder and CEO of Andarix Pharmaceuticals, which is using rhenium 188 P2045 (Tozaride) to target somatostatin receptor-bearing cells. The drug is currently in phase 2 for small-cell lung cancer.

Much of the action today, though, focuses on alpha particle emitters. "That's really the future," says Erik Mittra, a nuclear medicine specialist at the Oregon Health \& Science University in Portland. These isotopes, such as bismuth-213 and actinium-225, can release ten or more times as much energy as beta emitters, and the particles travel a tiny fraction of the distance, no further than about a few cell diameters. That means that "with good targeting," says
"You wind up being able to deliver higher radiation to tumors without exceeding any kind of safety limits."
So why is the trial using this lower dose? Because that's what doctors have always done, says Tagawa. Neither ABX nor Endocyte ever conducted a systematic dose-escalation study with 177Lu-PSMA-617.

Tagawa is also evaluating the potential of combining two different lutetium-177labeled PSMA-targeted agents for treating prostate cancer: 177Lu-PSMA-617 and a similar antibody-based therapy from Telix Pharmaceuticals of Melbourne, Australia. The hope, Tagawa says, is that the different circulatory dynamics and PSMA-binding preferences of the small peptide and large antibody carriers will complement each other.

Meanwhile, Bushnell and his colleagues at the University of Iowa's Holden Comprehensive Cancer Center are running a dual radioligand therapy trial of their own for patients with midgut neuroendocrine tumors. That trial involves two different beta-emitting isotopes Bruland, chief medical officer of Oncoinvent, "you can irradiate very small volumes of cancer without damaging normal tissues." The challenge remains overcoming target heterogeneity within cancers and controlling the 'recoil' energy of alpha decay, which can still yield daughter radionuclides that may do serious harm to healthy tissue. "It has to be handled very carefully," Mittra says.

Even with beta emitters, there are ways to deliver bigger radioactive payloads to the tumors. One approach involves simply upping the dose. Reporting in October at the European Society for Medical Oncology 2018 Congress in Munich, Germany, Scott Tagawa, a urologic oncologist at Weill Cornell Medicine in New York, showed that men with metastatic prostate cancer could tolerate a fractionated dose of 177Lu-PSMA-617 more than three times higher than what participants are getting in the phase 3 VISION trial. "And we could have gone higher," Tagawa notes, since his study reached its maximum planned escalation with no dose-limiting toxicities. tethered to two different tumor-targeting peptides. On the basis of a tumor's profile of norepinephrine transporters and somatostatin ticipants will receive customized doses of both an Azedra- and OctreoTher-like therapy. "You wind up being able to deliver higher radiation to tumors without exceeding any kind of safety limits," Bushnell explains. "It's a pretty slick little deal."

For Novartis, the decision to buy Endocyte largely boiled down to the company's radiopharmaceutical assets-177Lu-PSMA-617 and a preclinical variant with actinium-225 labeling of the same PSMA-targeted small molecule. But Endocyte has also been working on ways to make chimeric antigen receptor (CAR) T-cell therapies safer and more controllable. As the maker of Kymriah (tisagenlecleucel), the world's first approved CAR-T product (Nat. Biotechnol. 35, 691-693, 2017), Novartis could find that technology coming in handy.

Elie Dolgin Somerville, Massachusetts receptors, as revealed through imaging, par-

\section{RNAi biotechs flush with pharma dollars}

Two deals riding on the coattails of Alnylam Pharmaceuticals' first US approval for an RNA interference drug have seen billions flowing into the space. In October, Janssen Pharmaceuticals paid Arrowhead Pharmaceuticals $\$ 175$ million in cash for rights to an RNAi product designed to silence two regions in the hepatitis $B$ virus (HBV) genome, in a deal worth up to $\$ 1.6$ billion. The deal followed the release of striking interim results from a phase $1 / 2$ trial with the biotech's ARO-HBV (AROHBV1001) candidate. In the same month, Dicerna Pharmaceuticals received $\$ 100$ million up front and $\$ 100$ million in equity investment from Eli Lilly to use Dicerna's GalXC RNAi platform for generating oligonucleotide therapeutic agents in cardiometabolic disease, neurodegeneration and pain. Arrowhead's study in eight HBV-infected patients achieved a $96-99 \%$ drop in circulating HBV surface antigen in the groups treated with the two lowest doses of the drug. Chronic HBV infection can lead to cirrhosis and liver cancer. Standard of care requires long-term treatment with PEGylated interferons or oral nucleoside or nucleotide analogs, which stop viral replication.

The recent results are remarkable considering that in November 2016 Arrowhead's intravenous delivery vehicle for a second-generation RNAi drug was found to be toxic to nonhuman primates. The FDA put a stop on Arrowhead's phase 2 trial of the drug, and the company's stock plummeted. Arrowhead decided to abandon the programs and develop a new delivery technology, Targeted RNAi Molecule (TRiM), which they used to develop the candidates tested in the most recent trial. ARO-HBV contains two SiRNAs. Arrowhead was due to present more AROHBV1001 trial data at the Liver Meeting in November, as Nature Biotechnology went to press.

Dicerna also entered a deal with Alexion Pharmaceuticals in late October focused on the discovery and development of RNAi therapies to block the uncontrolled complement activation that drives many diseases. Dicerna received $\$ 22$ million up front and an equity investment of $\$ 15$ million, with potential for additional milestones and royalties.

Joana Osorio

\footnotetext{
66 The goal of the college is to "educate the bilinguals of the future." MIT president L. Rafael Reif coins the term 'bilinguals' for people in fields like biology, chemistry, politics, history and linguistics who are also versed in modern computing techniques, in his announcement of a $\$ 1$ billion college for artificial intelligence. (The New York Times, 15 October 2018)
}

\author{
"Science at this level is like a battleship, and it's \\ really hard to turn it around. People get emotionally \\ invested, financially invested, professionally \\ invested." Jonathan Moreno, a professor of \\ bioethics at the University of Pennsylvania, \\ speaking of the fall from grace of cardiac stem cell \\ researcher Piero Anversa. Some 31 research articles \\ on cardiac stem cells from Anversa's lab could be \\ retracted. (The New York Times, 29 October 2018)
}

"Some terms such as fake, synthetic and artificial
meat are not just intended to cast our products in
a negative light, they are also false and misleading.
We are making real meat and seafood, and that's
the whole point." Memphis Meats' Eric Schulze
says biotechs are adamantly opposed to the term
"lab-grown" while bemoaning the lack of new
regulation for labeling cell-based meats.
(Food Navigator, 25 October 2018) 